\title{
Análise das características físico-químicas de dois tratamentos de superfície em mini-implantes dentais
}

\author{
- Mariana Lima da Costa Valente Departamento de Materiais Dentários e Prótese, Faculdade de Odontologia de Ribeirão \\ Preto, Universidade de São Paulo, Ribeirão Preto, SP, Brasil • Diego Pedreira de Oliveira Departamento de Engenharia de \\ Materiais, Universidade Federal de São Carlos, São Carlos, SP, Brasil • Claudemiro Bolfarini Departamento de Engenharia de \\ Materiais, Universidade Federal de São Carlos, São Carlos, SP Brasil • Andréa Cândido dos Reis Departamento de Materiais \\ Dentários e Prótese, Faculdade de Odontologia de Ribeirão Preto, Universidade de São Paulo, Ribeirão Preto, SP, Brasil
}

RESUMO || Objetivos: A otimização das superfícies de titânio é essencial para acelerar o processo de osseointegração e viabilizar tratamentos reabilitadores em um curto período de tempo. O objetivo deste estudo foi avaliar comparativamente dois tratamentos de superfície do tipo nanométrico em mini-implantes dentários, sendo um comercial e outro experimental, quanto à caracterização morfológica e química. Materiais e métodos: Dois grupos foram avaliados: G1 - mini-implantes de titânio com tratamento de superfície Ossean ${ }^{\circledR}$ (Intra-Lock); e G2 - mini-implantes de titânio com tratamento de superfície experimental. Após o tratamento experimental, por meio de ataque com ácido fosfórico seguido de tratamento alcalino, os implantes foram avaliados por microscopia eletrônica de varredura (MEV), em dois modos diferentes: SE (elétron secundário), que analisa as alterações topográficas nas amostras, e BSE (elétron retroespalhado), que analisa as alterações ou flutuações de composição na superfície da amostra. A composição química foi verificada por um sistema de espectroscopia de energia dispersiva de raios X (EDS). Resultados: As imagens de MEV confirmaram diferenças nas superfícies G1 e G2, com presença de poros nanométricos no G2, enquanto a análise de EDS demonstrou a incorporação de elementos característicos da estimulação da neoformação óssea. Conclusões: O tratamento de superfície experimental, por ser um processo químico, além de simples, foi eficaz na formação de uma superfície rugosa e com capacidade bioativa.

DESCRITORES | Implantes dentais; Microscopia eletrônica de varredura; Osseointegração.

ABSTRACT | Analysis of physico-chemical characteristics of two surface treatments in dental mini-implants - Objectives: The optimization of titanium surfaces is essential to accelerate the process of osseointegration and to enable rehabilitative treatments in a short period of time. The objective of the present study was to evaluate comparatively two surface treatments of the nanometric type in dental mini-implants, being a commercial and another experimental, regarding the morphological and chemical characterization. Material and methods: Two groups were evaluated: G1 - titanium mini-implants with surface treatment Ossean ${ }^{\circledR}$ (Intra-Lock) and G2 - titanium mini-implants with experimental surface treatment. After the experimental treatment, by means of an attack with phosphoric acid followed by alkaline treatment, the implants were evaluated by scanning electron microscopy (SEM), in two different ways, SE (secondary electron) that analyzes the topographic changes in the samples and BSE (back-scattered electrons) that analyzes the composition changes or fluctuations in the sample surface. The chemical composition was analyzed by an X-ray dispersive energy spectroscopy (EDX) system. Results: SEM images confirmed differences in G1 and G2 surfaces, with the presence of nanometric pores in G2, whereas EDX analysis demonstrated the incorporation of elements characteristic of the stimulation of bone neoformation. Conclusions: The experimental surface treatment, as a chemical process, besides being simple, was effective in the formation of a rough surface and with bioactive capacity.

DESCRIPTORS | Dental implants; Scanning electron microscopy; Osseointegration.

AUTOR CORRESPONDENTE | - Andréa Cândido dos Reis Faculdade de Odontologia de Ribeirão Preto, Universidade de São Paulo • Av. do Café, s/n Ribeirão Preto, SP, Brasil • 14040-904 E-mail: andreare73@yahoo.com.br

- Received August 22, 2018 • Accepted August 27, 2018

- Dol http://dx.doi.org/10.11606/issn.2357-8041.clrd.2018.149226 


\section{INTRODUÇÃO}

O desenvolvimento dos tratamentos de superfície marcou uma nova era na implantodontia, cuja carga imediata após a instalação cirúrgica do implante foi a principal razão para os avanços tecnológicos na área. A microgeometria é fundamental para a estabilidade atemporal do implante no tecido ósseo ${ }^{1-3}$ e ainda representa um desafio para a indústria e pesquisadores, que buscam a devolução das funções fisiológicas e melhora da qualidade de vida dos pacientes, por meio de reabilitações orais em curto período de tempo..$^{3-4}$

Capazes de promover ações celulares e melhorar a integração com o osso circundante, os tratamentos de superfície favorecem maior atividade osteogênica, aceleram a osseointegração e viabilizam a realização de carregamentos precoces..$^{5-6}$ Três tipos de células estão envolvidas nesse processo: os osteoblastos, responsáveis pela osteogênese, as células epiteliais e os fibroblastos, que aderem ao componente transmucoso formando uma barreira e protegendo os tecidos ósseos subjacentes..$^{-9}$

Demonstrou-se experimentalmente que a rugosidade em escala micrométrica, promovida por procedimentos de jateamento de areia, ataque ácido, oxidação anódica e modificação com laser melhora e acelera a osseointegração, uma vez que aumenta a área de superfície e promove maior adesão de fibras colágenas, resultando em mais locais de ligação celular e crescimento tecidual. ${ }^{10-11}$ No entanto, a modificação da superfície também pode alterar o desempenho mecânico dos implantes, diminuindo a integridade estrutural e reduzindo significativamente a resistência à fadiga..$^{12-13}$

Tendências atuais demonstram que características superficiais em nanoescala apresentam potencial para favorecer respostas osteoblásticas ${ }^{3,14}$ e acelerar a osseointegração. Da perspectiva biomimética, uma nanoestrutura pode proporcionar uma topografia de superfície mais adequada para as funções celulares, uma vez que mimetiza melhor a estrutura dos tecidos ósseos, ${ }^{15}$ resultados estes comprovados por estudos de cultura celular in vitro. ${ }^{9}$

Alguns dos tratamentos de superfície em escala nanométrica comercialmente disponíveis apresentam componentes como cálcio, fosfato e cerâmicos em sua composição. Essa condição permite transformar a superfície de titânio e ligas lisas ou ligeiramente rugosas - portanto biologicamente inertes - para uma condição em que a bioativade é estimulada. Isso ocorre por meio de processos subtrativos seguidos de aditivos, como no caso do revestimento sol-gel, oxidação de micro arco, pulverização de plasma, deposição de laser pulsado e tratamentos químicos. ${ }^{16-17} \mathrm{O}$ principal motivo para essas associações é a promoção de aumento de rugosidade e elevada área superficial, que podem favorecer tanto a fixação mecânica quanto a adição de componentes importantes para a aderência de proteínas, essenciais durante o processo de cicatrização óssea. ${ }^{18-19}$

Embora diversos sejam os tratamentos de superfície disponíveis atualmente, o uso de técnicas mais simples, como processos químicos de combinação de ataque ácido com tratamento alcalino, também pode ser eficaz na promoção de equilíbrio entre propriedades mecânicas e biológicas, criando características micro e nanométricas na superfície do titânio. Assim, o objetivo deste estudo é avaliar comparativamente, por meio de caracterização físico-química, dois tratamentos de superfícies em mini-implantes dentários, um comercial e outro experimental, previamente testados in vitro.

\section{MATERIAL E MÉTODOS}

\section{Mini-implantes}

Para avaliar o tratamento de superfície foram utilizados dois modelos de mini-implantes dentários de titânio: G1 - mini-implante da Intra-Lock ${ }^{\circledR}$ System, com tratamento de superfície Ossean ${ }^{\circledR}$ (microjateado, com cálcio e fosfato a nível molecular, informações fornecidas pelo fabricante); e G2 
- mini-implante experimental, com tratamento de superfície nanométrico.

O mini-implante experimental, confeccionado em liga de titânio Grau V (Ti 6Al-4V), apresenta as mesmas dimensões do modelo comercial da IntraLock $^{\circledR}$ System (Flórida, Estados Unidos), MDL 2.010M, com Ø 2mm x $10 \mathrm{~mm}$ de comprimento, porém, com design diferente, semelhante à configuração de uma broca, com chanfros helicoidais de passo longo.

\section{Tratamento de superfície experimental}

Inicialmente, os mini-implantes experimentais foram lavados em aparelho de ultrassom UltraMet ${ }^{\circledR}$ 2003 Sonic Cleaner da Buehler (Chicago, EUA) durante sete minutos com água deionizada, seguidos de mais sete minutos com acetona, secos com jato de ar e armazenados em recipiente inerte até o momento da aplicação do tratamento de superfície. A modificação da superfície em escala nanométrica consistiu em ataque ácido, seguido de tratamento alcalino, de acordo com metodologia anterior. ${ }^{20-22}$

\section{Ataque ácido}

O ataque ácido foi realizado com ácido concentrado. A solução foi mantida à temperatura elevada, controlada com auxílio de um termômetro e conservada em placa de aquecimento MAo85 Marconi (Piracicaba, São Paulo, Brasil). Após a estabilização da temperatura, o mini-implante foi imerso na solução ácida e o béquer foi recoberto para reduzir a volatilização do reagente durante o procedimento. Na sequência, a amostra foi submetida ao tratamento alcalino.

\section{Tratamento alcalino}

O tratamento alcalino consistiu em preparar solução alcalina e transferi-la para um frasco de polietileno com tampa. A amostra atacada anteriormente com ácido foi mergulhada na solução alcalina. Uma estufa para secagem e esterilização MAo33 Marconi com ajuste de temperatura digital foi estabilizada a $60^{\circ} \mathrm{C}$, e o frasco contendo a amostra foi alocado no interior da estufa durante 24 horas.

\section{Caracterização físico-química dos mini-implantes}

A caracterização dos tratamentos de superfície, G1 e G2, foi realizada em cada um dos dois modelos de mini-implantes $(n=1)$, por meio de um microscópio eletrônico de varredura (MEV) Philips ${ }^{\circledR} \mathrm{XL}$ 30-FEG-SEM (Massachusetts, EUA), sob diferentes aumentos. A análise qualitativa da composição química ocorreu via espectroscopia com energia dispersiva de raios X (EDS) (Oxford Link ISIS 300), acoplado ao MEV. Foram obtidas imagens em dois modos diferentes: SE (elétron secundário), que analisa alterações topográficas nas amostras, e BSE (elétron retroespalhado), que analisa alterações ou flutuações de composição na superfície da amostra.

\section{RESULTADOS}

Os resultados foram direcionados para a caracterização da morfologia da superfície de ambos os implantes, a nível micro e submicrométrico. Aspectos da composição das superfícies também foram avaliados. Estudos prévios ${ }^{17,20-21}$ demonstraram que o tratamento de superfície utilizado no G2 promove aumentos nas rugosidades médias Ra e As, além disso, a elevada área de superfície associada à hidrofilicidade também foram reportadas. Por esse motivo, a reunião de tais características tornam interessantes sua investigação e comparação com tecnologias de tratamento de superfície vigentes no mercado odontológico.

\section{Modo SE (elétron secundário)}

Para o tratamento de superfície do grupo G1, do tipo microjateado (Ossean $囚$ ) analisaram-se os seguintes aumentos: $150 \times, 1.000 \times$ e $20.000 \times . \mathrm{Na}$ Figura 1 é possível observar em detalhes as macro e microcaracterísticas do implante e da superfície, com aumento da rugosidade da amostra devido ao processo de jateamento (Figura $1 \mathrm{~b}$ e 1c), que 
provocou a alteração de maneira desordenada, desde a escala macro até micro da superfície do implante.

Para o tratamento de superfície G2, realizaramse análises em aumentos de $150 \times, 1.000 \times \mathrm{e}$ $20.000 \times$. Devido à característica nanométrica evidente do tratamento aplicado, na Figura 2 podese observar a formação das microporosidades e nanotopografias.

Em maior aumento, de 100.000 vezes (Figura 3), é visível a diferença de tamanho dos poros presentes em G1 e G2, neste último, da ordem de nanômetros.

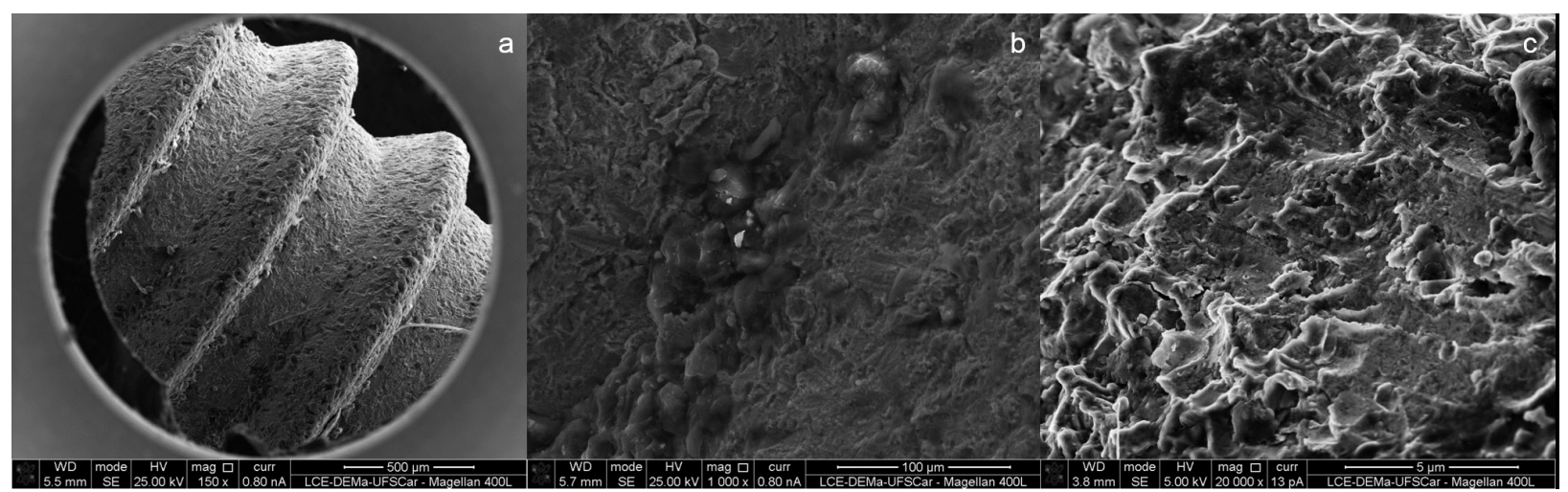

FIGURA 1 || Imagens do G1 no modo SE (elétron secundário) em aumentos de: a) 150 ×; b) 1.000 ×; c) 20.000 ×

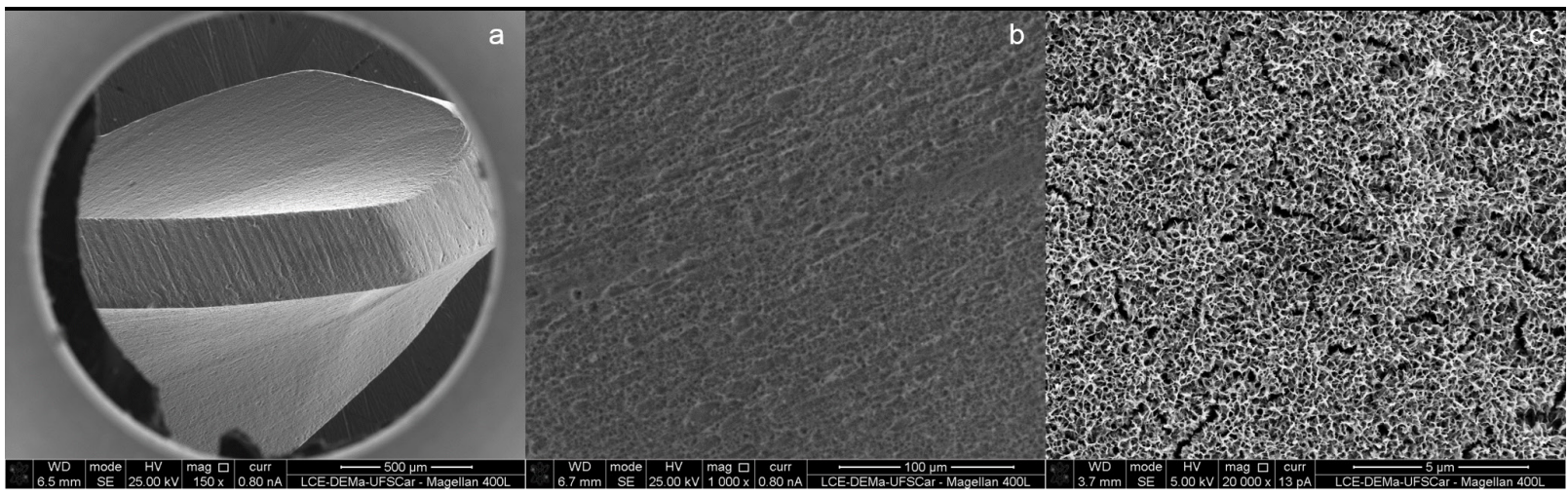

FIGURA 2 | Imagens do G2 no modo SE (elétron secundário) em aumentos de: a) 150 ×; b) 1.000 ×; c) 20.000 ×

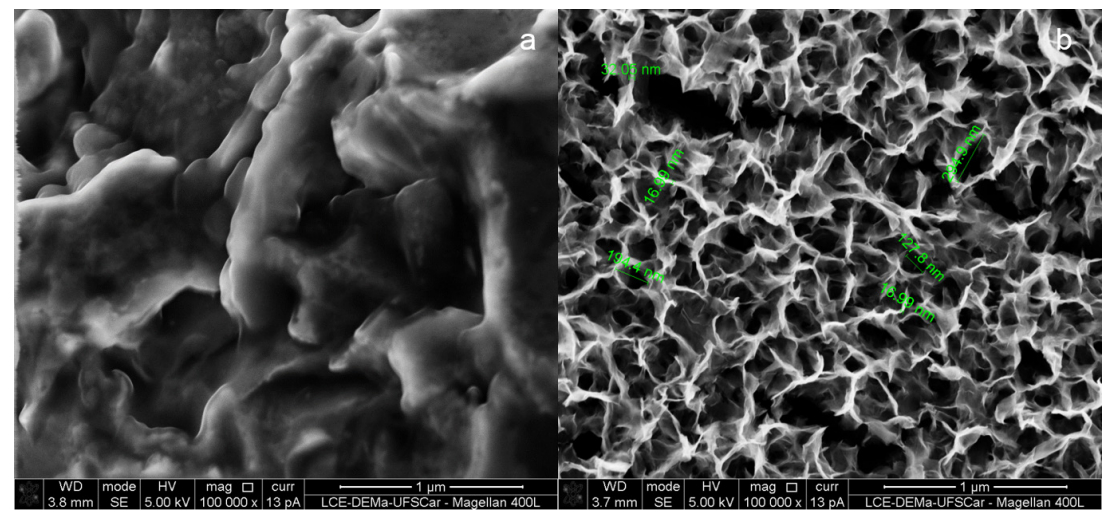

FIGURA 3 | Imagens das superfícies G1 e G2 no modo SE (elétron secundário) sob aumento de $100.000 \times$ : a) G1; b) G2 


\section{Modo BSE (elétron retroespalhado)}

Para o G1 é possível observar, na Figura 4, alterações de composições na superfície em que as regiões mais escuras se referem às maiores concentrações de elementos com menores massas

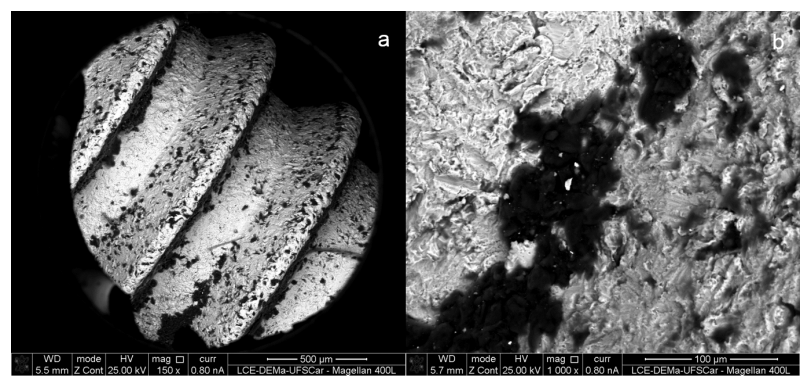

Figura 4 | Imagens do G1 no modo BSE (elétron retroespalhado) em aumentos de: a) $150 \times$; b) $1.000 \times$

\section{Composição química (EDS)}

A microanálise por espectroscopia com energia dispersiva de raios $\mathrm{X}$ (EDS) foi realizada em cada uma das amostras, no modo BSE (elétron retroespalhado), sob aumento de $10.000 \times$ (Figuras 6 atômicas, tais como $\mathrm{Cl}, \mathrm{Na}$ e $\mathrm{Ca}$, encontrados na microanálise da composição química via EDS.

Observa-se, na Figura 5, que o G2 alterou de maneira homogênea a superfície sem causar flutuações composicionais.

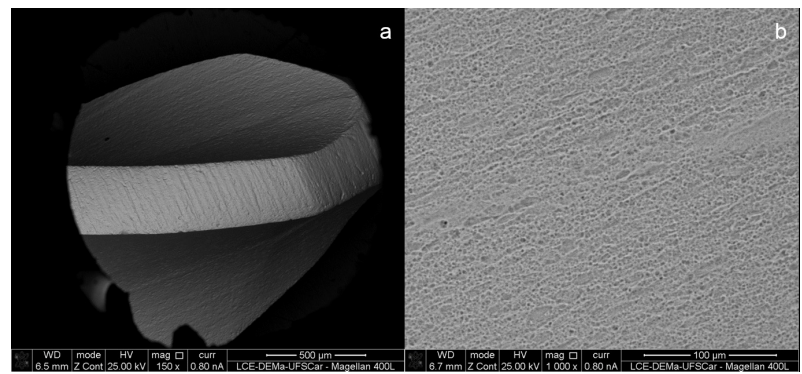

Figura 5 | Imagens do G2 no modo BSE (elétron retroespalhado) em aumentos de: a) $150 \times$; b) $1.000 \times$

e 7). O tratamento $\mathrm{G} 2$ revelou a presença de elementos nominais e contaminantes provenientes do ataque ácido, enquanto o tratamento alcalino causou a incorporação de sódio às superfícies e, inesperadamente, a incorporação do elemento cálcio.

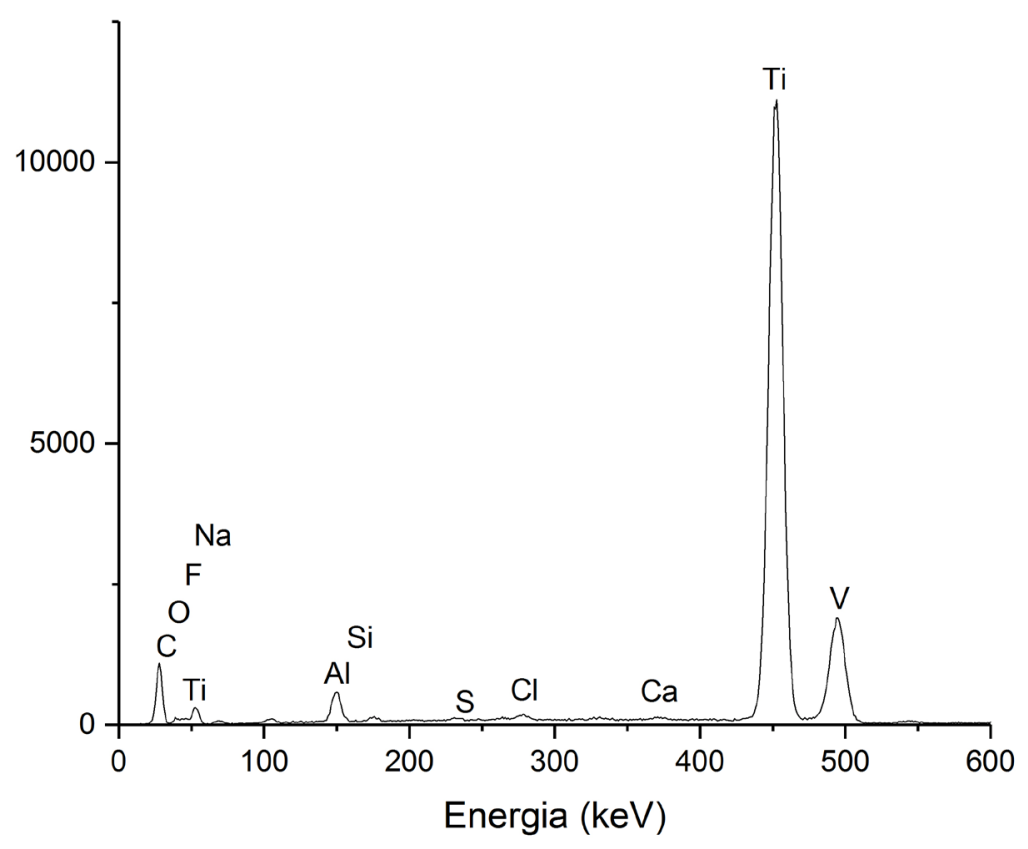

Figura 6 | Composição química (EDS) do G1 


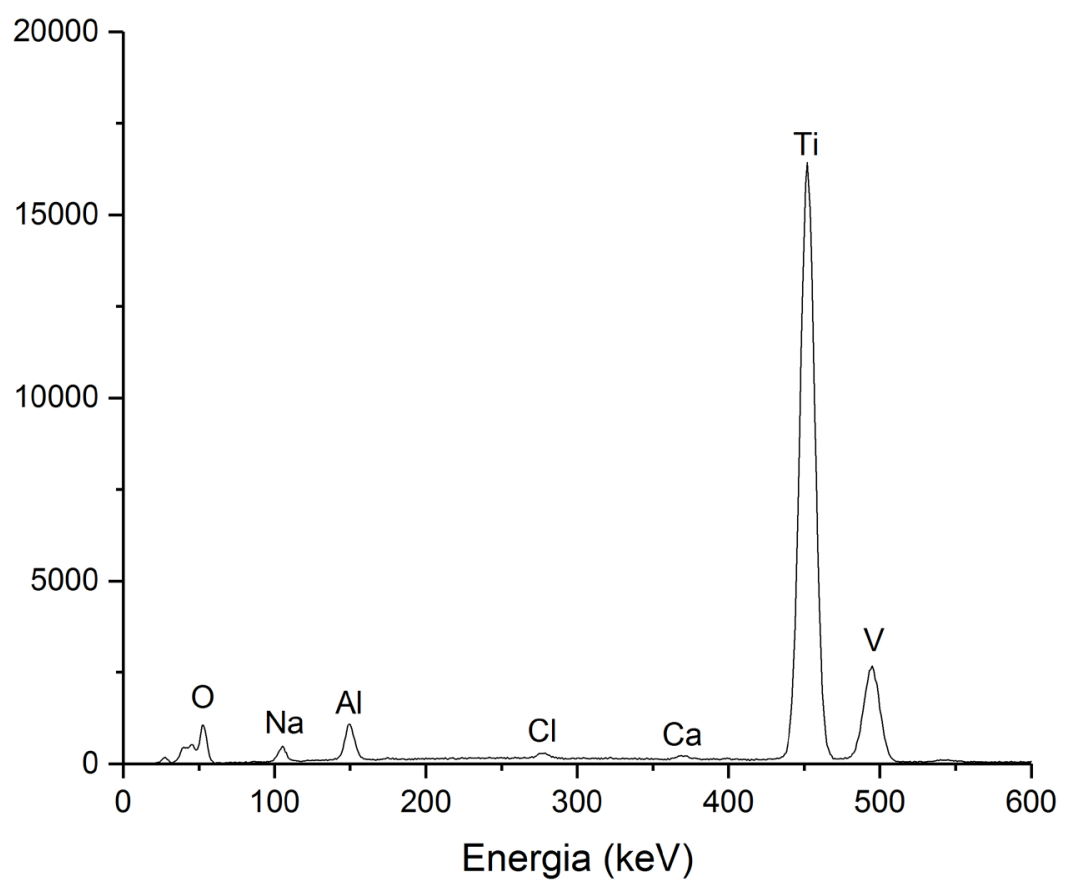

Figura 7 | Composição química (EDS) do G2

\section{DISCUSSÃO}

Alterações na superfície do implante são extensivamente investigadas, principalmente para aumentar a biocompatibilidade e osteocondutividade. ${ }^{23}$ Promover a bioatividade do titânio, por meio da incorporação de elementos como fósforo (P) e Cálcio (Ca), por exemplo, é fundamental para acelerar a precipitação de apatita na superfície, favorecer a adesão celular e respostas biológicas favoráveis ao processo de osseointegração. ${ }^{20,24}$

Os tratamentos de superfície podem ser obtidos por meio de tecnologias complexas ou métodos simples, tanto para modificar as superfícies como para aumentar a bioatividade do titânio. ${ }^{20}$ No estudo, a associação do tratamento com ácido fosfórico, responsável pela criação das microporosidades, e alcalino, por formar nanocaracterísticas que aceleram as respostas biológicas, promoveu a deposição de uma camada de titanato de sódio, sobre a qual a precipitação de hidroxiapatita é favorecida e catalisada importante fator para as respostas osteoblásticas. ${ }^{17,20,25}$
As imagens de MEV, produzidas no modo SE, mostraram que o tratamento ácido seguido de tratamento alcalino (G2) promoveu a formação de microporosidades e nanotopografias de maneira ordenada e uniforme. No G1, o aumento da rugosidade ocorreu de maneira desordenada, diferentemente do relatado pelo fabricante, segundo o qual cada implante é roboticamente microjateado por meio de um controle preciso, apresentando topografia de superfície semelhante em todos os níveis de ampliação, a nível nanométrico.

Além disso, no aumento de 100.000 vezes (Figura 3), observou-se diferença no tamanho dos poros formados e nas características superficiais conduzidas pelo tratamento de superfície experimental. Foram encontrados poros com dimensões abaixo de 1,0 micron no G2 e formação de estruturas da ordem de nanômetros, em comparação ao G1.

Algumas das superfícies de escala nanométrica comercialmente disponíveis apresentam componentes como cálcio, fosfato e cerâmicos que 
transformam a superfície de titânio biologicamente inerte em bioativa; isso, em associação com o aumento da rugosidade superficial, altera significativamente a resposta osso-implante nos tempos iniciais da implantação. ${ }^{23}$ Assim, o principal motivo para essas associações é promover rugosidade ótima, fixação mecânica e adicionar componentes importantes para a aderência de proteínas essenciais, durante o processo de cicatrização óssea. ${ }^{24}$

As imagens de MEV obtidas no modo BSE (elétron retroespalhado), que analisa alterações ou flutuações de composição na superfície/volume da amostra, associadas aos dados fornecidos pelo EDS mostraram a presença de componentes como cálcio e fosfato no G1, confirmando as informações fornecidas pelo fabricante. No G2, o tratamento alcalino também promoveu a incorporação de elementos como sódio, oxigênio e cálcio, como verificado em estudo realizado anteriormente. ${ }^{20}$ Tais componentes, assim como o $\mathrm{Mg}^{2+}, \mathrm{Sr}^{2+}$ e $\mathrm{PO}_{4}^{3-}$, estão presentes naturalmente no tecido mineral e apresentam importante papel na formação óssea..$^{26} \mathrm{O}$ que sugere a viabilidade do tratamento de superfície experimental utilizado no estudo.

Dentro das limitações in vitro deste estudo, podese dizer que o tratamento de superfície experimental avaliado é um método eficaz e simples para a modificação microgeométrica do titânio e sugere a formação de uma superfície bioativa, devido à presença de elementos como Ca. Além disso, esperase que a capacidade da superfície de absorver os componentes de cicatrização possa induzir a elevada taxa de recuperação em tempos menores para a osseointegração funcional do implante. Porém as respostas biológicas só poderão ser comprovadas com a realização de testes in vivo.

\section{CONCLUSÕES}

O tratamento de superfície experimental comparado ao comercial avaliado foi eficaz para formação de superfície com características nanométricas, além de apresentar elementos composicionais indicativos de bioatividade.

\section{AGRADECIMENTOS}

Agradecemos à Fundação de Amparo à Pesquisa do Estado de São Paulo (Fapesp), processo número 2014/27362-2, pelo financiamento dessa pesquisa.

\section{Conflito de Interesse: nenhum.}

\section{REFERÊNCIAS}

1. Bonfante EA, Granato R, Marin C, Jimbo R, Giro G, Suzuki $\mathrm{M}$, et al. Biomechanical testing of microblasted, acid-etched/ microblasted, anodized, and discrete crystalline deposition surfaces: an experimental study in beagle dogs. Int J Oral Maxillofac Implants. 2013 Feb;28(1):136-42. doi: 10.11607/jomi.2331.

2. Yeniyol S, Jimbo R, Marin C, Tovar N, Janal MN, Coelho PG. The effect of drilling speed on early bone healing to oral implants. Oral Surg Oral Med Oral Pathol Oral Radiol. 2013 Nov;116(5):550-5. doi: 10.1016/j.00oo.2013.07.001.

3. Coelho PG, Jimbo R, Tovar N, Bonfante EA. Osseointegration: hierarchical designing encompassing the macrometer, micrometer, and nanometer length scales. Dent Mater. 2015 Jan;31(1):37-52. doi: 10.1016/j.dental.2014.10.007.

4. Jimbo R, Tovar N, Marin C, Teixeira HS, Anchieta RB, Silveira LM, et al. The impact of a modified cutting flute implant design on osseointegration. Int J Oral Maxillofac Surg. 2014 Jul;43(7):883-8. doi: 10.1016/j.ijom.2014.01.016.

5. Stadlinger B, Pourmand P, Locher MC, Schulz MC. Systematic review of animal models for the study of implant integration, assessing the influence of material, surface and design. J Clin Periodontol. 2012 Feb;39(12):28-36. doi: 10.1111/j. 1600-051X.2011.01835.x.

6. Jeong SJ, Jeong MJ. Effect of Thymosin beta4 on the Differentiation and Mineralization of $\mathrm{MC}_{3} \mathrm{~T} 3-\mathrm{E} 1$ Cell on a Titanium Surface. J Nanosci Nanotechnol. 2016 Feb;16(2):1979-83.

7. Hamilton DW, Chehroudi B, Brunette DM. Comparative response of epithelial cells and osteoblasts to microfabricated tapered pit topographies in vitro and in vivo. Biomaterials. 2007 May;28(14):2281-93.

8. Kunzler TP, Drobek T, Schuler M, Spencer ND. Systematic study of osteoblast and fibroblast response to roughness by means of surface-morphology gradients. Biomaterials. 2007 Apr;28(13):2175-82. 
9. Miao X, Wang D, Xu L, Wang J, Zeng D, Lin S, et al. The response of human osteoblasts, epithelial cells, fibroblasts, macrophages and oral bacteria to nanostructured titanium surfaces: a systematic study. Int J Nanomedicine. 2017 Feb 20;12:1415-30. doi: 10.2147/IJN.S12676o.

10. Albrektsson T, Branemark PI, Hansson HA, Lindstrom J. Osseointegrated titanium implants. Requirements for ensuring a long-lasting, direct bone-to-implant anchorage in man. Acta Orthop Scand. 1981;52(2):155-70.

11. Velasco E, Monsalve-Guil L, Jimenez A, Ortiz I, Moreno-Muñoz J, Nuñez-Marquez E, et al. Importance of the roughness and residual stresses of dental implants on fatigue and osseointegration behavior. In vivo study in rabbits. J Oral Implantol. 2016 Dec;42(6):469-76. doi: 10.1563/aaid-joi-D-16-00088.

12. Leinenbach C, Eifler D. Influence of oxidation treatment on fatigue and fatigue-induced damage of commercially pure titanium. Acta Biomater. 2009 Sep;5(7):2810-9. doi: 10.1016/j. actbio.2009.03.029.

13. Pazos L, Corengia P, Svoboda H. Effect of surface treatments on the fatigue life of titanium for biomedical applications. J Mech Behav Biomed Mater. 2010 Aug;3(6):416-24. doi: 10.1016/j.jmbbm.2010.03.006.

14. Webster TJ, Ejiofor JU. Increased osteoblast adhesion on nanophase metals: $\mathrm{Ti}$, Ti6Al4V, and CoCrMo. Biomaterials. 2004 Aug;25(19):4731-9.

15. Liang J, Xu S, Shen M, Cheng B, Li Y, Liu X, et al. Osteogenic activity of titanium surfaces with hierarchical micro-/ nano-structures obtained by hydrofluoric acid treatment. Int J Nanomedicine. 2017 Feb 16;12:1317-28. doi: 10.2147/ IJN.S123930.

16. Le Guehennec L, Soueidan A, Layrolle P, Amouriq Y. Surface treatments of titanium dental implants for rapid osseointegration. Dent Mater. 2007 Jul;23(7):844-54.

17. Claros CAE, Oliveira DP, Campanelli LC, Pereira da Silva PSC, Bolfarini C. Fatigue behavior of Ti-6Al-4V alloy in saline solution with the surface modified at a micro and nanoscale by chemical treatment. Mater Sci Eng C Mater Biol Appl. 2016 Oct 1;67:425-32. doi: 10.1016/j.msec.2016.04.099.

18. Wennerberg A, Albrektsson T. Effects of titanium surface topography on bone integration: a systematic review. Clin Oral
Implants Res. 2009 Sep;20(Suppl 4):172-84. doi: 10.1111/j. 1600-0501.2009.01775.x.

19. Rosa MB, Albrektsson T, Francischone CE, Schwartz Filho HO, Wennerberg A. The influence of surface treatment on the implant roughness pattern. J Appl Oral Sci. 2012 Oct;20(5):550-5.

20. Oliveira DP, Palmieri A, Carinci F, Bolfarini C. Gene expression of human osteoblasts cells on chemically treated surfaces of Ti-6Al-4V-ELI. Mater Sci Eng C Mater Biol Appl. 2015 Jun;51:248-55. doi: 10.1016/j.msec.2015.03.011.

21. Oliveira DP, Prokofiev E, Sanches LFR, Polyakova V, Valiev RZ, Bottaa WJ. Surface chemical treatment of ultrafine-grained $\mathrm{Ti}-6 \mathrm{Al}-7 \mathrm{Nb}$ alloy processed by severe plastic deformation. J Alloys Compd. 2015 Sept;643(1):241-5. doi. org/10.1016/j.jallcom.2014.11.115.

22. De Oliveira BJS, Campanelli LC, de Oliveira DP, Guerra APB, Bolfarini C. Surface characterization and fatigue performance of a chemical-etched Ti-6Al-4V femoral stem for cementless hip arthroplasty. Surf Coat Technol. 2017 Jan;309:1126-34. doi.org/10.1016/j.surfcoat.2016.05.011.

23. Suzuki M, Guimaraes MV, Marin C, Granato R, Fernandes CA, Gil JN, et al. Histomorphologic and bone-to-implant contact evaluation of dual acid-etched and bioceramic grit-blasted implant surfaces: an experimental study in dogs. J Oral Maxillofac Surg. 2010 Aug;68(8):1877-83. doi: 10.1016/j. joms.2009.09.050.

24. Wennerberg A, Albrektsson T. Effects of titanium surface topography on bone integration: a systematic review. Clin Oral Implants Res. $2009 \operatorname{Sep} ; 20(4): 172-84$. doi: 10.1111/j. 1600-0501.2009.01775.x.

25. Bsat S, Yavari SA, Munsch M, Valstar ER, Zadpoor AA. Effect of Alkali-Acid-Heat Chemical Surface Treatment on Electron Beam Melted Porous Titanium and its apatite forming ability. Materials (Basel). 2015 Apr 8;8(4):1612-25. doi: 10.3390/ ma8041612.

26. Garcia-Gareta E, Hua J, Orera A, Kohli N, Knowles JC, Blunn GW. Biomimetic surface functionalization of clinically relevant metals used as orthopaedic and dental implants. Biomed Mater. 2017 Nov 22;13(1):015008. doi: 10.1088/1748-6o5X/aa87e6. 